\title{
L@s Otr@s de la Universidad Pública: Exclusiones y Desafíos que Persisten en Argentina
}

\section{The Others at the Public University: Exclusions and Challenges that Remain in Argentina}

\author{
Gabriela Bard Wigdor ${ }^{1 *}$ \\ Gabriela C. Artazo ${ }^{2}$ \\ 1 Universidad Nacional de Córdoba \\ 2 Consejo Nacional de Investigaciones Científicas y Técnicas (CONICET) de Argentina
}

\begin{abstract}
A partir de investigaciones teóricas previas y del trabajo de campo con sectores populares, arribamos a la conclusión de que amplios grupos sociales considerados "los otros" por la hegemonía del saber-poder, es decir, quienes no son varones blancos de sectores medios y altos, experimentan procesos de exclusión de las IES. En el presente artículo, reflexionamos sobre estos procesos en la Universidad pública de Argentina, sobre todo en la provincia de Córdoba. Proponemos una introducción sobre la génesis colonial de la Universidad y sobre su rol histórico. Luego, reflexionamos sobre el enfoque epistemológico y pedagógico que guía el artículo, un diagnóstico sobre la situación actual de la Universidad y posibles políticas de inclusión de cada vez más ciudadanos/as, que apuesten a la construcción de un conocimiento participativo, inclusivo, multicultural y democrático. Concluimos que la Universidad es una institución inseparable del modelo de Estado y del clima político de la época.
\end{abstract}

Descriptores: Universidad pública, Sectores populares, Género, Estado, Colonialidad.

From previous theoretical research and field work with popular sectors, we came to the conclusion that large social groups considered "the others" by the hegemony of knowledge-power, are expelled. This sector named "the others" are not white males of middle and high class. These sectors are experimenting excluding processes. In this article, we try to reflect these processes at the public University of Argentina, especially in the province of Cordoba. We propose an introduction from the colonial genesis of the University and its historical role. Then we explained the epistemological and pedagogical approach that guides the article, a diagnosis of the current situation of the University and inclusion policies. Analyzing the policies that are committed to building a participatory, inclusive, multicultural knowledge and democratic. Concluding that the University is an institution that can't be discuss without the political time and model of state.

Keywords: Public university, Popular sectors, Gender, State, Colonialism.

*Contacto: gabrielabardw@gmail.com

ISSN: 0718-7378

www.rinace.net/rlei/
Recibido: 16/06/2016

$1^{\text {a }}$ Evaluación: 28/07/2016

Aceptado: 20/09/2016 


\section{Introducción}

Partimos de la constatación empírica, a partir de nuestras investigaciones previas en sectores populares cordobeses ${ }^{1}$, de que la universidad pública y gratuita en la Argentina, aún con todos los avances logrados hasta el año 2015, como fue el ingreso irrestricto a cualquier carrera de grado, tutorías para ingresantes, becas para financiamiento de materiales de estudios, comedor universitario gratuito para becados/as ${ }^{2}$ y al precio de 30 centavos de dólar para el resto de la comunidad educativa, transporte público subsidiado para estudiantes, etc. continúa siendo una institución excluyente o que incluye según los intereses de las relaciones de saber-poder hegemónicas en el sistema colonial capitalista y patriarcal.

En ese sentido, recordemos que la universidad es una institución que nace a partir de la instauración en el año 1492 de la relación colonial-moderna, que inicia con el genocidio de los pueblos indígenas de América. La modernidad que trajo Europa, se erige sobre la explotación económica y la dominación cultural de las sociedades americanas (Quijano, 2014). La universidad no escapa a las instituciones que surgieron en las regiones periféricas, con la mirada puesta en la Europa occidental moderna y en las últimas décadas en la norteamericana.

En la provincia de Córdoba, la creación de la universidad se remonta al año 1613, producto de la política de ocupación colonial española. Los dirigentes que ocupaban las funciones de mando eran españoles de origen y era tarea de las universidades la preparación de los funcionarios de bajo nivel de responsabilidad de gobierno. No ingresaban a la Alta Casa de Estudios representantes de pueblos originarios, mulatos o zambos, mujeres y tampoco criollos americanos. La educación era impartida a grupos reducidos de personas que conformaban la elite económica, política y cultural de la época.

La universidad reproducía el perfil educativo de las universidades españolas, caracterizadas por su impronta escolástica y religiosa. El tipo de formación se caracterizaba por su contenido religioso, siendo la fe católica doctrina política del imperio español y por intermedio de ella, los dirigentes y funcionarios de las colonias

\footnotetext{
${ }^{1}$ Las investigaciones en la cuales se origina este escrito responden; por un lado, a una investigación empírica concluida denominada: "Culturas políticas de mujeres de sectores populares: políticas desde lo cotidiano”, realizada para obtener el título máximo de Doctora en Estudios de Género (Bard Wigdor, 2015), financiada por CONICET. En esta investigación se trabaja con mujeres de sectores populares, indagando en sus trayectorias políticas, donde la profundización acerca de sus recorridos educativos formales, cobra una relevancia analítica indiscutible para analizar posiciones sociales. Por otro lado, la otra investigación en curso "Estado, Instituciones y Políticas Públicas" (Artazo, 2015), analiza la incidencia del Programa Nacional Progresar, como parte de la estrategias de reproducción social de jóvenes insertos/as en unidades educativas formales de una ciudad de Córdoba (Villa María). El objetivo del plan investigativo es indagar si el programa inciden o no en la re-conversión de capitales de dichos/as jóvenes. Además, investigar la percepción del adulto/a referente sobre los/as jóvenes-que participan en dicho programa. Financiado por CONICET, la investigación se desarrolla en el marco del Doctorado en Cs. Políticas del CEA-UNC.

2 A lo largo del artículo utilizaremos "lenguaje inclusivo" como estrategia narrativa para destacar el género del sujeto y evitar un lenguaje que universalice desde lo masculino. Debido a que el lenguaje influye poderosamente en las actitudes, el comportamiento y las percepciones de las personas sobre el resto y sobre sí mismas, y bajo la premisa de que "lo que no se nombra no existe", solicitamos comprensión ante el uso de este estilo que tal vez resulte algo incómodo para la lectura, pero representa una apuesta política relevante para quienes no somos el sujeto universal de la palabra: las mujeres. En ese sentido, la lengua ha sido usada desde hace siglos como el soporte de las estrategias patriarcales de subordinación de las mujeres y otros géneros, por lo que lleva inscriptas y ocultas relaciones de poder. Sostiene opciones léxicas sesgadas, discriminatorias o degradantes al implicar que los varones son superiores al resto. Por ejemplo, en el uso de lo masculino como lo universal, como si fuera el modo de incluir y nombrarnos a todas/os. El género masculino se usa como "inclusivo", mientras que el femenino es "exclusivo", es decir, que se refiere solamente a las mujeres.
} 
asimilaron la visión del mundo de la potencia metropolitana (Recalde, 2010). Para Mignolo (2014), la universidad y el museo fueron las dos instituciones fundamentales en la construcción y gestión de "la colonialidad del saber".

En ese sentido, la universidad es en primer término producto de un proceso que como sostiene Quijano (2000), comenzó con la constitución de América y del capitalismo colonial/moderno como patrón de poder mundial. De hecho, América se fundó en este nuevo patrón de poder mundial, donde la codificación de las diferencias entre los conquistadores y los pueblos originarios fue la idea de raza, es decir, "una supuesta diferente estructura biológica que ubicaba a los unos en situación natural de inferioridad respecto de los otros" (Quijano, 2000, p. 246). Los colonizadores codificaron como color los rasgos fenotípicos de los pueblos indígenas y a partir de esta característica, asociaron todo tipo de supuestas inferioridades intelectuales, morales, etc. No se los consideraba personas, mucho menos sujetos de conocimiento.

Con el transcurso de las décadas y la instauración del Estado Nación en Argentina, propiamente con la Revolución de Mayo, el logro de la independencia de España y la sanción de la Constitución Nacional en el año 1853, se iniciaron cambios relevantes en la Argentina, aunque la Universidad era una institución que se resistía con empeño a cambiar su perfil colonial y dependiente.

En la segunda mitad del siglo XIX y comienzos del siglo XX, se sanciona en Argentina el "sufragio universal"s (Ley Sáenz Peña, 1912) y nacen movimientos políticos de gran trascendencia como el movimiento universitario reformista. En Córdoba, en esa misma época, la Universidad Nacional sostenía lógicas de la época colonial, donde la investigación científica era casi inexistente, al igual que en las otras Universidades de Latinoamérica. Enfrentando esta situación, se fortalecen los movimientos sociales que pretendían democratizar el acceso a la universidad. En Córdoba, especialmente importante fue la Reforma Universitaria de 1918, que denunciaba el oscurantismo, el autoritarismo y el elitismo en la universidad y cuyos logros fueron: la modificación de los planes de estudio, la democratización de la enseñanza, la participación de los estudiantes en el gobierno universitario, la libertad de pensamiento y los derechos políticos. Desde esta reforma, las universidades nacionales adquirieron el carácter de autónomas, aunque continúan, dependiendo -aún hoy- del presupuesto que le asigna el Estado nacional (autárquicas).

Otro momento importante en la historia Argentina para la Universidad, fue cuando se decreta la gratuidad de los servicios universitarios, establecida por decisión del primer peronismo4 en el año 1949. Esto produjo la ampliación del ingreso, donde hijos/as de trabajadores accedían masivamente a estudios de grado. Existe abundante bibliografía que indica que la educación como medio de movilidad social e integración se instala con anterioridad en la sociedad Argentina (Sarlo, 2001; Tedesco, 2000; entre otros autores), siendo la reforma universitaria de 1918 un emergente de esas nuevas presiones sociales. Sin embargo, durante el primer peronismo esta idea se consolidad y se crean

\footnotetext{
s "Sufragio Universal" es una formulación sexista debido al carácter sesgado y performativo de esta formulación discursiva, que universaliza una política que sólo alcanzaba a los varones y sólo a un sector privilegiado de ellos (Barrancos, 2005; Valobra, 2008; entre muchas otras investigaciones).

${ }^{4}$ Se denomina primer peronismo al gobierno de Juan Domingo Perón comprendido entre el año 1946 hasta las elecciones generales de 1952, en las que volvió a ganar Perón por un 52\% de los votos.
} 
instituciones de gran relevancia social como la universidad obrera de 1948 y el Consejo Nacional de Investigaciones Técnicas y Científicas (CONICET) en 1951.

Durante las décadas subsiguientes al primer peronismo y hasta la década del 60, la universidad argentina fue modelo de excelencia académica, en la que se destacaron notables científicos/as de reconocimiento mundial. Pese a esto, la década del 60' sobrelleva un proceso de autoritarismo y violencia en el cual se produce "la Noche de los Bastones Largos" ${ }^{\circ}$ en junio de 1966 y se inauguran los años de dictadura y genocidio en nuestro país.

Cabe recordar que los dispositivos educativos propios de las sociedades modernas, tal como lo es la Universidad, construyeron y reprodujeron sentidos que sedimentaron en el sentido común de las grandes mayorías. No solo en términos de transferencia de conocimiento sino que el modo en que esta; expulsó o incluyó sectores poblacionales, investigo y formo generaciones, delimitó un ámbito de lo posible dentro de este espacio educativo superior. Esta constitución intrínseca relacional de la Universidad posibilitó que esta fuera protagonista de momentos de cambio como así también de represión. Durante las dictaduras militares la universidad vivió etapas oscuras, de violencia estatal y silencio. Muchos/as científicos/as se fueron del país, inaugurando la llamada "fuga de cerebros". Poco se produjo en materia de conocimiento crítico, en un escenario social atravesado por la desaparición de docentes y alumnos/as de las diferentes carreras, tildados/as de "subversivos" por la dictadura del momento (1976). Estos regímenes dictatoriales desaparecieron generaciones de jóvenes militantes, intelectuales críticos y sindicalistas (Martinetto, 2008). Genocidio que tuvo por objetivo incorporar las políticas económicas del Neoliberalismo en Latinoamérica y educar a las sociedades en valores antidemocráticos y colonialistas

En la década del 80, con la vuelta a la Democracia, en Argentina se reabrieron carreras que habían sido clausuradas por considerárselas subversivas, se extendió la cobertura educativa y el restablecimiento de los principios declarados por la Reforma Universitaria: "significantes como democracia, participación, pluralismo, soberanía, entre otros, eran adoptados como el marco desde el cuál se enunciaban diferentes proyectos educacionales" (Martinetto, 2008, p. sd).

Para la década de los 90, el gobierno sancionó dos leyes relevantes para la Universidad: la Ley de Reforma del Estado y la Ley de Emergencia Económica. Estos instrumentos legales se orientaron a la conformación de un nuevo modelo de Estado. Este abandona su papel de interventor en la regulación de la economía y en la redistribución de la riqueza, para favorecer la libertad de mercado y satisfacer las demandas de los sectores de mayor concentración de capital (Richmond, 2006). Se requería la formación de egresados/as cuyas subjetividades contribuyeran a consolidar y desarrollar el modelo neoliberal. Como sostiene Richmond (2006), esto implicaba sujetos competitivos, individualistas, apolíticos y eficientes. La pobreza en producción intelectual crítica fue evidente en este periodo, donde la profesionalización según la demanda del mercado fue el eje de la formación universitaria.

\footnotetext{
${ }^{5}$ Se denomina "Noche de los bastones blancos" a la represión por parte del Estado del 29 de julio de 1966 hacia cinco facultades de la Universidad de Buenos Aires (UBA), ocupadas por estudiantes, profesores y graduados, en oposición a la decisión del gobierno militar de intervenir las universidades y anular el régimen de gobierno.
} 
Durante esta etapa se sanciona en 1995 la Ley de Educación Superior (LES), que dejaba libre y a criterio de cada facultad, las maneras de acceso de los y las alumnos/as a los estudios superiores. Se permitió el arancelamiento y los cursillos de ingreso eliminatorios. Debido a un fuerte descontento social frente a este tipo de políticas, se crearon nuevas universidades públicas y privadas como la Universidad Nacional de Quilmes, UNTREF, San Martín, UNGS, entre algunas, que paradójicamente, dieron acceso a importantes sectores de la población tradicionalmente excluidos de la universidad. Estas universidades, organizadas con modelos flexibles, se dirigieron a absorber parte de la demanda que recibía la Universidad de Buenos Aires. Esto posibilito también, colocar direcciones afines al gobierno en las direcciones universitarias, de manera de obtener mayoría en el Consejo de Universidades (Marquina, 2011).

Finalmente, en este breve recorrido histórico, debemos mencionar los últimos 12 años (2003-2015) del país, que diferentes autores (Adamovsky, 2012; Kessler, 2014; Merklen, 2010; entre otros/as) caracterizan como "la vuelta a un Estado social", que se inicia en el año 2003 y marca un tiempo universitario de grandes mejoras, entre las cuales: se aumenta el presupuesto universitario, el cual pasó del $2 \%$ al 6,5\% del PIB (durante el período 2003-2015) como meta de la ley de financiamiento educativo aprobado por el congreso, aumentan un $843 \%$ los salarios del sector; se implementó un programa de infraestructura universitaria único, que incluyó la realización de obras con una inversión de más de 1382 millones de pesos. A su vez, se crearon y abrieron universidades en las localidades populares del Gran Buenos Aires, Avellaneda, Florencio Varela, Moreno, Merlo y José C. Paz, en Villa Mercedes (San Luis), Río Negro y en Tierra del Fuego. Actualmente todas las provincias cuentan, por lo menos, con una Casa de Altos Estudios. Un dato a destacar es que una gran cantidad de los alumnos/as de esas nuevas universidades es la primera generación de universitarios en su familia y que desde el INDEC se señala que desde 1991 a 2013 , la población de mujeres que accedió a la universidad pasó de 44,6 \% a 57, 6\% (Chiroleu y Marquina, 2015).

Otro dato relevante es que la Universidad Nacional de Córdoba, se convirtió en la primera Casa de Altos Estudios de Argentina en contemplar en su reglamentación el reconocimiento de la identidad de género elegida. Se declara "institución libre de discriminación por identidad y expresión de género" y gracias a esta normativa, los/as miembros de la comunidad universitaria podrán ser reconocidos por su identidad elegida, aunque ésta no sea la registrada en la documentación formal; para tal fin presentan una nota con carácter de declaración jurada.

Por medidas como las descriptas, podemos afirmar que la Universidad se ha democratizado con el correr de los siglos, aunque esto no signifique que haya abandonado totalmente su carácter de institución colonial, que perpetúa, aunque de un modo más sutil, desigualdades raciales, clasistas y de género. Encubre bajo un discurso de diversidad, el fortalecimiento de la idea del logro individual, propio del liberalismo capitalista. Es el individuo "quien debe superarse" para ingresar a la universidad y sobre todo para permanecer. Como sostiene Fuentes Vásquez (2015):

Es más, en un sentido estricto la formación para el reconocimiento y el respeto de las diferencias, y el logro efectivo de una mayor igualdad de oportunidades -para las mujeres, las personas con desventajas socioeconómicas, con identidades $y$ orientaciones sexuales diversas y los grupos étnicos, entre otros- no ha sido una prioridad para las agendas académicas de las instituciones de educación superior. (p. 46) 
A continuación, nos adentramos en el enfoque epistemológico que guía el análisis propuesto en este artículo, partiendo metodológicamente de los datos arrojados por el trabajo de campo que las autoras han realizado en diferentes comunidades populares de Córdoba, y a partir del análisis y recuperación de aportes teóricos de otros/as autores/as que resultan relevantes. Luego, desarrollamos un diagnóstico sobre la situación de la Universidad en la Argentina y en Córdoba, para cerrar con algunas reflexiones sobre el desarrollo de políticas de inclusión y democratización de la Universidad.

\section{Posición epistemológica: el porqué de la inclusión}

Dentro de las ciencias y de la producción de conocimiento en general, ha sido prolifero el debate en torno a la neutralidad de la ciencia en términos epistémicos y de su técnica en términos de aplicación. Hay quienes sostienen que si bien la ciencia no es neutra, debe tener cierto grado de objetividad que permita someter las hipótesis de trabajo a la evidencia empírica dentro de un proceso de investigación, lo que conduciría a conclusiones "verdaderas o no" sobre lo que se estudia.

Al respecto, se encuentra ampliamente discutido y consensuado como una ingenuidad, afirmar que pueden existir verdades inocuas que se "revelan" mediante la constatación empírica como fuerza irrefutable. Si esto fuera así, dotaríamos a la realidad de una acción propia, desligada de los agentes que la producen mediante prácticas sociales -por medio de las cuales- se configura la dialéctica histórica del devenir social.

Por tanto, el planteo reflexivo que aquí expondremos no intenta establecerse como "verdad", sino que pretende fundamentar la vital importancia que tiene la inclusión de las grandes mayorías de la población a la formación de grado superior, asumiendo para ello, una posición política y subjetiva clara. En tal sentido, aspiramos a desarrollar y promover dentro de las IES una política educativa tendiente a equiparar oportunidades, reconocer diferencias y posicionarlas como usinas generadoras de diálogos multiculturales (desde un pensamiento crítico), en el marco de la exigibilidad del derecho a la educación.

Es por esto que consideramos como prioritario la necesidad de construir marcos interpretativos sobre dichos problemas, que puedan polemizar con aquellos planteos que dentro de la teoría de la política pública, toman como indicadores de una performance gubernamental eficiente sólo el ingreso, permanencia y graduación de los/las estudiantes de las IES. Estas miradas tecnocráticas -con su supuesto planteo "neutral"son los fundamentos principales a la hora de realizar recortes presupuestarios y de cupo dentro de las universidades, sin tomar en cuenta la compleja configuración de los//las estudiantes que se constituyen en agentes activos dentro de este campo y la existencia de significativas diferentes posiciones de los/as agentes, a partir de una estructuración del volumen y posesión de capitales desiguales, que influyen en estos procesos de ingreso y egreso. Olvidando evaluar las acciones de inclusión e exclusión de las propias instituciones educativas.

Asimismo, al predominar una perspectiva monetarista y eficientista sobre la educación formal en los análisis de las políticas públicas, se reafirman presupuestos "naturalizados" sobre el "gasto excesivo" que sería sostener determinadas carreras de grado, como aquellas que no son fuertemente demandadas por el mercado, especialmente las que se consideran de escasa aplicación técnica, abonando el paradigma de la educación como 
gasto y no como inversión social, y la concepción de la educación superior como servicio y no como derecho.

Para confrontar con este enfoque, coincidimos con Lander (1997) en que la búsqueda de alternativas a esta sociedad excluyente y desigual, amerita esfuerzos de deconstrucción de perspectivas positivistas en las ciencias, que bajo el supuesto carácter universal y natural de su enfoque, reproducen y ofrecen argumentos para consolidar la sociedad patriarcal, capitalista y liberal. Se requiere urgentemente del cuestionamiento de las pretensiones de objetividad y neutralidad de los principales instrumentos de naturalización y legitimación de este orden social: el conocimiento científico moderno y las instituciones que lo producen.

Para Viveros Vigoya (2009), el racismo y el sexismo de los enfoques científicos dominantes, naturalizan la diferencia y la desigualdad social, argumentando que las relaciones sociales son biológicas y basadas en las diferencias fenotípicas (como el color de piel). A su vez, ambos asocian el dato corporal con la realidad social y caracterizan a los grupos que no son dominantes como predispuestos a la sumisión. Asimismo, crean supuestos universales acerca de las experiencias de los/as subalternos, como la utilización de la categoría mujer sobre mujeres, para ocultar las diversas experiencias y modos culturales de experimentar el género, la clase, etc.

Por tanto, para analizar las posiciones que ocupan los diferentes agentes sociales en la estructura universitaria y fuera de ella, sin reproducir estos enfoques universalizadores, el enfoque interseccional nos propone atender al género, la clase, la etnia, la nacionalidad, entre otras posiciones, y a las relaciones que se establecen entre estas dimensiones como articulaciones entre sistemas de opresión. Nacido desde el movimiento de mujeres negras y elaboradas como teoría formal por una de sus referentes Kimberlé Crenshaw (1995), el enfoque define la interseccionalidad como la expresión de un "sistema complejo de estructuras y opresión que son múltiples y simultáneas" (Crenshaw, 1995, p. 359). Es la consecuencia de factores de discriminación que interactúan con otros mecanismos de opresión "creando una nueva dimensión de desempoderamiento" (Crenshaw, 1995, p. 359).

Vazquez Laba (2008) sostiene que la interseccionalidad como estrategia de análisis, es uno de los modos que dispusieron las feministas para abordar las diferencias entre las mujeres, modo que tiene presente las geografías, historias y las diversidades culturales. Así, se analiza la diferencia sexual en relación a la etnia, al estilo de vida, a la religión, a la preferencia sexual y a la nacionalidad.

Con este planteo no negamos que, como sostiene Haraway (1999), no existan puntos claves de lucha o conflictos claves desde donde impulsar cambios, como puede ser el género para los movimientos feministas. Sucede que no debemos comprometernos con una única categoría de análisis, sino que desde este enfoque, debemos pensar la relación de intersección entre categorías y no a ellas como categorías que construyen pares binarios, por ejemplo, blanca/negra o burgués/trabajador. "La separación categorial es la separación de categorías que son inseparables” (Lugones, 2008, p. 76).

La interseccionalidad atiende a la relación entre raza/etnicidad/clase/sexualidad para dar cuenta de experiencias diferenciadas, tal como plantea el feminismo del punto de vista y las teorías pos y decoloniales. De este modo, atendemos a la condición de las/os inmigrantes, a la etnicidad cuando confronta con la idea de nacionalidad y ciudadano de 
una nación. También, a la generación, a la edad y a la clase cuando interactúan y estructuran las formas de organización social. Miramos cómo operan en diferentes niveles de la vida cotidiana con sus múltiples expresiones en lo personal, lo simbólico y lo institucional.

Este modo de analizar la realidad y los conflictos sociales, nos permite indagar en las exclusiones y desigualdades que atraviesan las instituciones educativas, integrando el género, la raza, la clase, como intersecciones fundamentales que hacen a la cuestión social, reduciendo las visibles omisiones que reproducen las políticas sociales cuando toman como sujeto de intervención a quienes han sido históricamente el sujeto del conocimiento y de la ciudadanía en general: el varón blanco y burgués.

\section{Qué sucede con la universidad del siglo XXI en Córdoba, Argentina}

Hernández Arregui (1972) sostiene que todo análisis de la labor intelectual de una época, deberá atender a las siguientes dimensiones: la clase social, la dimensión psicológica de todo acto creador, la conformación sociológica de los ámbitos de producción, la acción de las clases dominantes (tanto internas como externas) que luchan por ganar el sentido y la práctica de los/as intelectuales, la realidad con su articulación histórica concreta.

Desde esta perspectiva, comprendemos que los sectores populares del campo y de la ciudad tienen grandes dificultades para ingresar y permanecer en las instituciones educativas formales, resultado de su posición histórica en la estructura social, la cual condiciona determinada calidad de vida, y de acuerdo al carácter clasista de las IES, que no garantizan trayectorias educativas exitosas para todo el conjunto social.

En tal sentido, las comunidades con las que hemos trabajado para nuestras investigaciones doctorales, en un porcentaje que ronda casi el 90\% de jóvenes y mujeres entrevistadas (sobre una población de 90 entrevistas), no han finalizado el nivel educativo secundario e incluso cuentan con el primario incompleto. Sus hijos/as no escapan a la dinámica expulsiva de las instituciones formales, encontrándose por fuera del sistema educativo medio. De hecho, el secundario es una gran incógnita en la Argentina, comprender por qué los y las jóvenes de sectores populares encuentran marcadas dificultades para ingresar y permanecer sigue siendo un problema clave.

Por tanto, se deduce que mayores dificultades encuentran estos sectores para acceder a la educación superior. La Universidad es una institución prácticamente inexistente en el imaginario de estos sectores, porque no existe en el universo de lo posible transitar una carrera de grado. Quien logra acceder a la Casa de Altos Estudios es considerado o considerada una rareza para su entorno y cuenta con mayor capital social y económico que la mayoría de las familias de su comunidad. Suele deberse, en los casos donde hemos investigado, a hijos/as de padres y madres obreros/as, que supieron estar incluidos a través del empleo, obtuvieron título secundario y valoran el estudio como oportunidad de ascenso y progreso social. Son sectores medios empobrecidos, que en los 90 se denominaban "nuevos pobres". En el resto de los casos con los que trabajamos, son generaciones de sujetos excluidos del empleo formal y la educación, predominando el primario incompleto y en varias ocasiones, dificultades para leer y escribir. 
Dentro de estas comunidades, las mujeres son quienes antes se ven excluidas del trayecto educativo formal. Desde la infancia deben ocuparse de "las tareas del cuidado": cuidar a sus hermanas/os menores, ayudar a limpiar la casa, cargar con las tareas domésticas que su madre no pueda afrontar, etc. El peso de las desigualdades de género es evidente y visible a diario, cuando caminamos por las calles de las diferentes comunidades populares y observamos mujeres jóvenes sentadas en la puerta de su casa, barriendo, atendiendo hermanos/as, hijos/as, en horario donde deberían poder concurrir a la escuela. Estudiar es sin duda, un privilegio de clase.

El fenómeno de feminización de la pobreza se expresa en variados indicadores, entre ellos los referidos a educación, dan cuenta de un tipo de exclusión que recorta oportunidades en términos de integración social desde el sistema educativo formal. La expulsión de las instituciones educativas superiores adopta complejos mecanismos de distinción que se constituyen como parte de trayectorias educativas diferenciadas por la posición de clase. Debido a esto -y a la acumulación y apropiación diferenciada de volúmenes de capitales de los agentes- en el momento de ingreso a la universidad, aquel/la sujeto/a que mantuvo pretensiones de ingreso a la misma, encontrará variados obstáculos propio de un campo en el cual los procesos de distinción son intrínsecos a la constitución del mismo. Ingresante mujer madre de 7 hijos, con secundario completo finalizado a distancia y sin empleo formal, es el "fenotipo" de una distinción negativa de quienes no podrá permanecer y formarse como profesional ya que la universidad no se amoldará a sus necesidades -y a pesar de los enunciados esfuerzos de inclusión que ha tenido la universidad- las exigencias y capacidades que exige la permanencia le indicaran a la agente que su lugar no es dentro sino fuera. Con esto queremos ejemplificar el modo sutil e inocuo con el que las instituciones educativas formales superiores quedan fuera del imaginario de aquellos sectores subalternos- que por posibilidades del contexto inmediato- arriesgan un posible ingreso a la misma. Simplemente se retiran del campo universitario con el sedimento naturalizado de que esto "no es para ellos/as", las IES quedan fuera del horizonte de sentido de los/as agentes.

Por otro lado, retomando los planteos de Hernández Arregui (1972), si queremos comprender las problemáticas de inclusión de estos sectores por parte de la Universidad, es necesario atender a la conformación sociológica del campo donde actúan los y las agentes que la constituyen. La Universidad es un centro de producción de conocimiento, de formación de intelectuales y profesionales, que tiende a la complicidad y reproducción de relaciones de dominación al interior de la clases sociales del país y del conjunto de países del centro capitalista sobre la periferia, a partir de la aculturación, apoyada por los poderes económicos y los medios de comunicación de masas. Como sostiene Bauer (2001) esto se concreta sin la necesidad, como fuera en otras épocas, de ocupación territorial permanente y sin bandera, pero ejerciendo presiones externas económicas, políticas y culturales.

En relación a la aculturación, afirma Darcy Ribeiro (1971) que la actual configuración étnica de la humanidad “(...) es el resultado del exterminio de millares de etnias, de la fusión de razas y de la difusión lingüística y cultural" (p. sd). La dominación cultural apunta a que todos los sectores sociales adopten el modo burgués de mirar el mundo, constriñe el pensamiento a la llamada civilización occidental: un sentido común capitalista. Las Universidades para Ribeiro (1971) actúan como agentes de conservación del status quo y en definitiva son fruto y productoras de la sociedad, para cambiarlas, la sociedad debe cambiar junto a ellas. 
Por otro lado, analizando las relaciones sociológicas del campo específico de la Universidad, donde se reproducen las lógicas de dominación que intervienen a nivel estructural, lo que se encuentra en juego, en primera instancia, es el saber específico y calificado, sumado a una titulación que nos habilite como portadores de un saber específico (la dimensión profesionalizante de la universidad). Para disputar este capital en juego, los y las agentes ponen en acto diversas estrategias de reproducción social (Bourdieu, 1993), siendo estas un conjunto de prácticas fenomenológicamente muy diferentes, por medio de las cuales estos/as tienden de manera consciente o inconsciente a conservar o a aumentar su patrimonio y correlativamente, a mantener o mejorar su posición en la estructura de las relaciones de clase.

La capacidad de producción y reproducción de estrategias de los/as agentes, son desiguales de acuerdo a las posiciones disimiles que cada cual ocupa en el espacio social que trasciende el campo educativo y que responde a la trayectoria de vida de cada agente, definida en parte por lo que su grupo primario de pertenencia y lo que la sociedad pudo ofrecerle en términos de capital económico, social, simbólico y cultural.

Para sustentar empíricamente estos análisis, tomamos los cursos de ingresantes a las carreras de la Universidad pública y gratuita de Córdoba, donde las autoras somos profesoras y constatamos que efectivamente, los y las agentes se encuentran ubicados/as en posiciones diversas y desiguales tales como: jóvenes de sectores medios, jóvenes de sectores populares, mujeres de comunidades originarias, varones homosexuales, mujeres madres, entre tantas otras intersecciones. Esta diversidad de posiciones presentan interrogantes de todo tipo, entre los cuales: ¿podrán las mujeres sortear el cursillo de ingreso con éxito, cuando muchas de ellas son madres y deben concurrir con sus hijos/as al aula?; ¿las mujeres de comunidades originarias, se sentirán interpeladas y familiarizadas con la cultura vigente en esta institución?; ¿quiénes se encuentran provistos de los habitus ${ }^{6}$ necesarios para permanecer en la institución?; ¿quiénes acceden y quienes son expulsados/as incluso antes de ingresar a la Universidad por la cultura institucional y por los recursos económicos que demanda el simple hecho de asistir?, entre tantas otras.

Partiendo de que la academia y sobre todo en las IES, se construyen espacios sociales que tienden a legitimar de modo predominante un conocimiento de rasgos eurocéntrico, positivista, patriarcal y clasista, considerando que todavía hoy las diversas y desiguales culturas y sujetos del mundo que no tienen la representación proporcional en la historia universal (Bauer, 2001), los interrogantes parecen no responderse favorablemente para las posibles trayectorias educativas de estas/os ingresantes.

El modo en que se configuran los regímenes de enseñanza y las prácticas educativas dan cuenta, como sostendría Freire (2002), de relaciones de opresión en el vínculo con el/la educando, desdeñando otros modos de aprehender y de enseñar, uniformando los saberes y legitimando la figura de un tipo específico de educando que puede ingresar y permanecer: el varón de sectores sociales medios y altos. No predomina el respeto por los intereses y la multiplicidad de experiencias y saberes de los/as educandos que deciden comenzar con una formación de grado superior. Esta situación podría

${ }^{6}$ Es uno de los conceptos centrales de la teoría sociológica de Pierre Bourdieu (1993). Refiere a esquemas de obrar, pensar
y sentir asociados a la posición social. El habitus hace que personas de un entorno social homogéneo tiendan a compartir y sentir asociados a la posición social. El habitus hace que personas de un entorno social homogéneo tiendan a compartir estilos de vida parecidos. 
encuadrarse en lo que llamábamos, junto a Grignon y Passeron (1979) como arbitrariedad cultural, la cual precisa para su despliegue un cumulo de acciones dirigidas expulsar, excluir a los educandos que no se ciñan a esta.

Para tal propósito el sistema educativo superior argentino posee los más refinados mecanismos de violencia simbólica ${ }^{7}$, donde no se agrede ni excluyen directamente a los/las educandos, sino que se va recortando sus posibilidades de permanencia y persistencia por medio de complejos mecanismos de distinción, en donde solo aquellos/as con disposiciones u habitus dominantes podrán sostener y adaptarse a los mismos.

Los/as educandos que ingresan de modo "marginal" a los ámbitos educativos de la IES, perciben ciertamente que el mundo que allí se les presenta les es ajeno, cumpliéndose la premisa de la clásicas teorías educativas modernas de la "tabula rassa", las cuales suponen a los/las educandos como nulos de saber, siendo la "transferencia" educativa el modo en el que vehiculizan el proceso de enseñanza aprendizaje (llenar el recipiente vacío). Esta transferencia educativa va configurar ciertas prácticas dentro de las IES que pretenderán mediante el velo de la evaluación y calidad educativa, legitimar a la cultura dominante para consolidar las posiciones de aquellos educandos homologados por sus posiciones dentro de la misma.

En ese sentido, toda cultura académica es arbitraria, puesto que su validez proviene casi exclusivamente de la cultura de las clases dominantes, legitimadas ante la totalidad de la sociedad como evidente saber objetivo. Esto al interior de las IES se ve fortalecido por currículas académicas y perfiles profesionales tecnocráticos, acríticos y de escaso debate acerca del aporte que debieran realizar los y las universitarios/as al desarrollo de su país y en relación a las necesidades de las grande mayorías sociales. Además, no existe planificación estatal acerca de que sectores estratégicos de la universidad debieran desarrollarse, que perfiles precisa la sociedad y que recursos calificados demanda el ámbito público independientemente del mercado.

La Universidad pública debiera responder a las necesidades del ámbito público, del cual dependen la mayoría de la población no privilegiada, y no de las demandas del ámbito privado, como actualmente sucede en la gran mayoría de las carreras de grado. Al respecto, Ernesto Guevara (1959) señalaba ya en la década del 50 respecto a la educación cubana, que era el gobierno quien debía planificar el desarrollo industrial y productivo del país, por tanto, tenía necesidad y derecho a fijar las características y la cantidad de egresados/as que precisaría en un futuro para satisfacer necesidades de la Nación.

Actualmente, el Estado Cubano pauta y demanda la formación de médicos, deportistas, ingenieros, etc. según las necesidades del desarrollo de su país y de su población. Lo cual no desmerece que él o la sujeto puede elegir formarse en una carrera que considere afín a sus intereses, sino que propone repensar la educación pública, como un derecho que depende del Estado para ser garantizado y que demanda que este sea soberano, solidario y eficaz en lo público. Características que no pueden lograrse si continuamos formando en la Universidad pública, profesionales que responden a los intereses y demandas del mercado, para lo cual, existen las ofertas de las Universidades privadas. Es el mercado quien debe responder económicamente y a través de la creación de sus propias

${ }^{7}$ Definiremos «violencia simbólica» como la acción pedagógica que impone significaciones y las impone como legítimas (Bourdieu, 1993). 
estructuras educativas por los "recursos humanos" que precisa. El Estado, en representación de la población argentina, no puede ni debe financiar la formación de profesionales que no ofrecerán sus conocimientos para el desarrollo del país y que incluso, como hemos explicados, trabajan en la consolidación de su situación mundial periférica, su empobrecimiento y desigualdad.

En tal sentido, la educación superior argentina enfrenta no sólo problemáticas históricas de formación de profesionales con poca o escasa conciencia nacional, sino políticas de exclusión racista, por género y clase; sumado a nuevos desafíos derivados del liberalismo y que tienen que ver con la masificación, la expansión y la especialización del conocimiento. Como respuesta se apostó por la diferenciación institucional y la diversificación de carreras, lo que incidió en el aumento de la complejidad de la universidad. No hay planificación estatal en la materia y por tanto, el Estado tampoco asegura que el mercado absorba a todos/as sus alumnos/as cuando logran egresarse. A demás de estos conflictos, entre los desafíos actuales de inclusión, vuelve a discutirse viejos presupuestos reificados en el sentido común que precisan de una batalla cultural incesante, como son destronar del podio de la naturalidad el supuesto de que sólo determinados agentes ingresan y permanecen en las IES por estar "más capacitados/as" (las teorías meritocráticas) o porque "hay quienes son para la pala y otros para el libro" (Darwinismo social).

Por lo tanto, si lo que se quiere es promover la inclusión de múltiples agentes, pertenecientes a distintos sectores sociales, culturales, de género, etc. Es preciso reflexionar y accionar sobre varias dimensiones que hacen a la universidad: en el aspecto estructural que mencionábamos, es decir, en la planificación y en el perfil político general de la educación pública, y en el aspecto cualitativo de los encuadres de enseñanza, además de vehiculizar políticas de inclusión según las necesidades que plantean las intersecciones que atraviesan la vida de los y las sujeto

\section{El carácter patriarcal de la sociedad que la universidad reproduce}

Las mujeres están accediendo masivamente a los estudios superiores, logran licenciarse y algunas incluso ser reconocidas alumnas destacadas de sus carreras. Según López Cleip y Amoroso (2007), a partir de datos de la Coordinadora de Investigaciones e Información Estadística (CIIE) de la Secretaría de Políticas Universitarias del Ministerio de Educación de la Nación y de la Secretaría de Políticas Universitarias, el porcentaje de mujeres en la matrícula estudiantil de las Universidades Nacionales Argentinas pasó de un $44,6 \%$ a $57,6 \%$ en 2007.

La llamada feminización de la educación viene a confirmar que cuanto más central es un ámbito para la sociedad, cuanto más poderoso es un grupo, tanto menos están representadas las mujeres (Beck, 1998, citado por Marrero, 2006, p. 49). Para Beck (1998), ellas sólo consiguen conquistar "barcos que se hunden" o que están en riesgo de hacerlo (Beck, 1998, citado por Marrero, 2006, p. 49). En ese sentido, en las distintas regiones, las mujeres suelen estar más fuertemente educadas que los varones, obtienen licenciaturas y maestrías en mayor proporción, según estudios de la Unesco. De hecho, los títulos de doctorado están repartidos por igual entre hombres y mujeres, y aunque la mayor escolarización en estudios superiores se encuentra entre las mujeres, no garantiza 
que lleguen a cargos altos en empresas ni en ámbitos públicos, como parte del fenómeno que las feministas denominan techos de cristal $^{8}$.

De hecho, en el mundo, el 87 por ciento de las juntas directivas de empresas están presididas por hombres y sólo el 13 por ciento por mujeres, según el informe de la Organización Internacional del Trabajo (OIT). El llamado "techo de cristal" con el que chocan las mujeres a la hora de acceder a puestos de responsabilidad, sigue siendo un problema vigente que la Universidad no discute ni acciona para su modificación. Para las estudiantes, trabajadoras no docentes y alumnas de la Universidad, resulta difícil conciliar el mundo laboral y el mundo familiar, ya que se les exige igual que a los varones o más en mismos puestos de trabajo y de formación, mientras en la familia siguen siendo demandadas como el principal sostén organizativo y emocional (Bard Wigdor y Borchi, 2014).

Asimismo, las mujeres no sólo enfrentan discriminaciones en los lugares de poder y de formación, una investigación sobre trayectorias laborales docentes de las mujeres en la Universidad de Córdoba a cargo de Rodigou, Blanes y Domínguez (2011), muestra que en el ambiente laboral existen situaciones de discriminación, maltrato y violencia por razones de género. Las autoras identificaron situaciones en las que mujeres, gays, lesbianas, transexuales, travestis fueron sujetas de maltratos y violencias. Estas situaciones afectan la construcción de un proyecto laboral y en ocasiones, condicionan la permanencia en la propia institución universitaria.

En ese sentido, la manera en que se estructura la academia tiene diversas implicancias en el desarrollo de la vida de las mujeres que participan y en quienes quedan fuera. Quienes acceden deben cumplir con una serie de tareas meritocráticas, inspiradas en la vida cotidiana de los varones, a la cual pueden acceder las mujeres, pero a partir de sacrificios de todo tipo, como relegar la vida personal y aceptar como condición, esforzarse el doble que los varones para obtener iguales o peores resultados. Se vuelve habitual e incluso natural la explotación de las mujeres de sí mismas y de otras, en una competencia despiadada por los pocos espacios de poder que se les ofrecen. Esta situación se hace explícita y evidente por ejemplo, en las licencias de maternidad que se les otorgan a las mujeres en al ámbito científico, donde los plazos para la producción de conocimiento son apremiantes (como artículos o ponencias), en la asistencia indefectible a los lugares de trabajo, en la competitividad que se espera que desempeñen más allá de sus condiciones de producción. Indudablemente no es un problema exclusivo de la academia, pero ésta lo refuerza y lo legitima.

En ese orden, el sistema universitario y científico se estructura para mujeres que se espera no sean madres o no tengan cargas familiares (enfermos y discapacitados), o en su defecto, detenten posiciones socioeconómicas que respondan a estratos altos y medios. Situación económica que les permite costear los gastos de que terceras se ocupen de este tipo de tareas (casi siempre mujeres), consideras como privadas por el sistema sexual de división del trabajo. En este sentido, podríamos afirmar que la percepción dominante es que la mujer "puede" salir a efectuar trabajo productivo fuera del hogar, siempre que siga reproduciendo correctamente su rol original en la familia, o en su defecto, abandone totalmente su desarrollo en estas áreas. De modo que el ambiente de la Universidad y de

\footnotetext{
${ }^{8}$ Se denomina techo de cristal a la limitación velada del ascenso laboral de las mujeres al interior de las organizaciones. Se
} trata de un techo que limita sus carreras profesionales, difícil de traspasar y que les impide seguir avanzando. 
la producción científica no está aún en condiciones, pese a los avances en materia de género, para incluir y mantener a la diversidad de mujeres, mucho menos otros géneros (Borchi y Bard Wigdor, 2014).

Por otra parte, la equidad de género no proviene únicamente del sistema universitario y científico, mientras las mujeres continúen ocupándose del amplísimo abanico de responsabilidades que toman a su cargo, aun teniendo las mismas posibilidades y derechos en el plano formal, no podrán afrontar los requerimientos permanentes del mundo académico y laboral en general. Este tipo de problemáticas no son abordadas como debieran por el mundo académico, pues como dice Nava Flores y Aguilar (2009):

\title{
(...) en lugar de dedicar tiempo y esfuerzo en el diseño de políticas que apunten al corazón de las situaciones que las mujeres más están sufriendo, las políticas públicas y sus elaboradores, se distraen en la consideración de temas que poco importan a las mujeres y que resultan extraños a sus intereses. (p. 75)
}

Finalmente no podemos desconocer que la exclusión de las mujeres de los lugares de poder, como de tantos/as otras/os sujetos no varones, responde a una serialización de quienes se considera capaces de administrar, investigar y dirigir, aquellos quienes representan el ideal de sujeto que produce conocimiento: el varón blanco y heterosexual. Quedan por fuera del sistema problemáticas y necesidades de una pluralidad de sujetos que no se consideran en la Universidad ni en la ciencia en general, y/o no tienen lugar para representarse. Como dice Castoriadis (1975) si no se le da visibilidad al sujeto, si no es reconocido/a, no existe, es nada.

\section{Reflexiones sobre la Universidad como escenario de oportunidades, diversidad y diálogos multiculturales}

\author{
Qué la universidad se pinte de negro, que se pinte de mulato, no sólo entre los \\ alumnos, sino también entre los profesores, que se pinte de obrero y de campesino, que \\ se pinte de pueblo, porque la Universidad no es el patrimonio de nadie y pertenece al \\ pueblo .... \\ La Universidad debe ser flexible, pintarse de negro, de mulato, de obrero, de \\ campesino, o quedarse sin puertas, y el pueblo la romperá y él pintará la \\ Universidad con los colores que le parezca. (Che Guevara)
}

Para abordar el desafío vital de instituir espacios en la Universidad que permitan construir un proyecto pluriversal que acompañe a los/as estudiantes para que comprendan, denuncien y trabajen en contrarrestar la colonialidad del poder en su cotidianeidad y en sus cuerpos, es necesario como mínimo, que la currícula de la universidad plantee desde el ingreso el debate acerca de qué universidad para qué modelo de país y de región.

En ese sentido, la universidad debería discutir y difundir una nueva versión de la historia, negada por la ciencia positivista occidental, aquella que visibiliza a los pueblos originarios, a las culturas latinoamericanas y que dialoga profundamente con las necesidades y demandas de sus poblaciones. Aquel conocimiento que bregar por desarrollos productivos inclusivos y respetuosos del paisaje, que propone modificaciones en el enfoque colonial y eurocéntrico predominante, así como en el carácter sexista del conocimiento que se difunde de manera hegemónica. 
En este desafío, es innegable que entre las dificultades que se presentan para la construcción de pensamiento crítico, el neoliberalismo debatido y confrontado únicamente como teoría económica es una. En realidad, el neoliberalismo debe ser comprendido como el discurso hegemónico de un modelo civilizatorio, es decir, un conjunto de supuestos y valores básicos de la sociedad liberal moderna en torno a la sociedad, la riqueza, la naturaleza, la historia, etc. (Lander, 1997).

En relación con el perfil neoliberal de los/as profesionales, debemos cuestionarnos el carácter únicamente profesionalizante que está adquiriendo la universidad, cuando esta no sólo debería transmitir saberes que habilitan para el ejercicio profesional y especializado, sino que ser una escuela de pensamiento, de formación crítica en lo que concierne a la realidad social. Recuperar su lugar de generación de pensamiento crítico, de intelectuales comprometidos/as con su época y los problemas de la misma es urgente.

Asimismo, ampliar sus bases a los sectores sociales que son excluidos en razón de su género, clase o color. Para lo cual, no es suficiente con que se dicten materias, cursos, taller sobre pensamiento crítico, género y diversidad sexual, tienen que acceder a las aulas educandos y educadores de diferentes etnias, género y sectores sociales. Tiene que ser posible transexuales en las aulas, mujeres que masivamente ocupen cargos jerárquicos como docentes titulares, personas de color, indígenas, etc. que puedan enseñar su versión de la historia y legitimar sus demandas y necesidades sociales. Eso sería efectivamente políticas dirigidas a pluralizar el conocimiento y el o la sujeto que conoce.

Además, tal como señalara Freire, la educación no puede continuar siendo una mera transmisión de información digerida y cómplice con la visión de los sectores dominantes. La universidad debe afrontar procesos colectivos de construcción de conocimiento, donde no queden por fuera experiencias y saberes que no son los legitimados por la ciencia positivista. Hablamos de los saberes que provienen de la praxis y de la trasmisión generacional, tan vivo en los pueblos originarios y en las mujeres en lo que concierne a lo mal llamado privado: la familia, la crianza, etc. Esos espacios son escenarios de aprendizaje y laboratorios de conocimiento que debieran encontrar canales para poder expresarse en las instituciones formales.

Asimismo, insistimos en que la universidad debe discutir el modelo de país al que pretende contribuir, porque de ese modelo efectivamente depende la universidad que se tiene y que se puede llegar a tener. Para que los sectores populares accedan al estudio superior, para que se democratice el público universitario, el Estado debe estar presente para garantizar los derechos sociales fundamentales de los/as ciudadanos/as. Un pueblo que no come, que no accede a la salud pública, al ejercicio político, difícilmente pueda educarse.

\section{Referencias}

Adamovsky, E. (2012). Historia de las clases populares. Desde 1880 hasta 2003. Buenos Aires: Sudamericana.

Bard Wigdor, G. (2015). Culturas políticas de mujeres de sectores populares: políticas desde lo cotidiano (Tesis doctoral). Universidad Nacional de Córdoba. 
Bard Wigdor, G. y Borchi, A. (2014). La productividad científica en el sistema capitalista: una mirada feminista y decolonial. En P. Peyloubet (Comp.), Reflexiones y experiencias situadas. Una contribución a la pluralización de conocimientos (pp. 230-260). Buenos Aires: Ed. Nobuko.

Bauer, F. (2001). Crítica a la concepción eurocentrista. Recuperado de http://www.manuelugarte.org/

Bourdieu, P. (1993). Sociology in question. Londres: Sage Publications.

Castoriadis, C. (1975). La institución imaginaria de la sociedad. Barcelona: Tusquets Editores.

Freire, P. (2002). Pedagogía de la esperanza. Buenos Aires: Siglo XXI

Fuentes Vásquez, L. (2015). Inclusión, equidad y mercado: ¿objetivos irreconciliables de la educación superior? Revista NÓMADAS, 44(4), 45-67.

Grignon, C. y Passeron, J. C. (1979). Lo culto y lo popular. Miserabilismo y populismo en sociología y literatura. Buenos Aires: Nueva Visión.

Hernández Arregui, J. (1972). Imperialismo y cultura. Buenos Aires: Plus Ultra.

Kessler, G. (2014). Controversias sobre la desigualdad. Argentina 2003-2013. Buenos Aires: Fondo de la Cultura Económica.

Laba Vazquez, V. (2008). Las contribuciones del feminismo poscolonial a los estudios de género: interseccionalidad, racismo y mujeres subalternas. Revista del Instituto Cubano de Investigación Cultural Juan Marinello, 1(3), 46-77.

Lander, E. (1997). Modernidad, colonialidad, postmodernidad. Revista Venezolana de Economía y Ciencias Sociales, 3(4), 11-28.

López Cleip, B. y Amorozo, T. (enero, 2007). Feminización de la matrícula universitaria. Ponencia presentada en el $V$ Encuentro nacional latinoamericano la universidad como objeto de investigación. Universidad Nacional del Centro de Buenos Aires.

Lugones, M. (2008). Colonialidad y género. Tabula Rasa, 5(9), 73-101.

Marquina, M. (2011). El ingreso a la universidad a partir de la reforma de los '90: las nuevas universidades del conurbano bonaerense. En N. Gluz (Comp.), Admisión a la universidad y selectividad social (pp. 63-86). Buenos Aires: Editorial de la Universidad Nacional de General Sarmiento.

Marquina M. y Chiroleu, A. (2015). ¿Hacia un nuevo mapa universitario? La ampliación de la oferta y la inclusión como temas de agenda de gobierno en Argentina. Revista Propuesta Educativa, 43(1), 7-16.

Martinetto, A. (2008). La década del 80 en la universidad pública. Normalización post-dictadura y transición hacia la reforma de los años 90.Valencia: Mimeo.

Merklen, D. (2010). Pobre ciudadanos. Las clases populares en la era democrática. Buenos Aires: Gedisa.

Mignolo, W. (2014). Los desafíos decoloniales de nuestros días: pensar en colectivo. Neuquén: Universidad Nacional del Comahue.

Nava Flores, C. y Aguilar, R. (2009). La explicación psicosocial de la violencia hacia las mujeres en Miahuatlán, una alternativa para el diseño de políticas de igualdad de género. Disponible en: http://www.hacienda.go.cr/

Página 12. (2015). Quedan paredes y techos de cristal. Página 12, 13 de enero. Disponible en: http://www.pagina12.com.ar/diario/sociedad/3-263789-2015-01-13.html (13/01/2016) 
Quijano, A. (2000). Cuestiones y horizontes: de la dependencia histórico-estructural a la colonialidad/descolonialidad del poder. Buenos Aires: CLACSO.

Recalde, A. (2010). La universidad argentina, del modelo colonial al reformismo. Recuperado de http://sociologia-tercermundo.blogspot.com.ar/

Ribeiro, D. (1971). La universidad latinoamericana. Caracas: Ediciones de la Biblioteca de la Universidad Central de Venezuela.

Richmond, I. (2006). La mercantilización de la universidad pública argentina: políticas nacionales de articulación de los dos subsistemas de la educación superior. Revista Española de Educación Comparada, 12, 407-428.

Rodigou, M., Blanes P. y Domínguez, A. (2011). Territorios y Fronteras de Género en la Universidad Nacional de Córdoba. Espacios en Blanco. Revista de Educación, 23(1), 73-97.

Sarlo, B. (2001). Ya nada será igual. Punto de Vista, 70(24), 2-12.

Tedesco, J. C. (2000). Educar en la sociedad del conocimiento. Buenos Aires: Fondo de Cultura Económica.

Valobra, A. (2008). La ciudadanía política de las mujeres y las elecciones de 1951. Anuario del Instituto de Historia Argentina, 8, 53-89.

Viveros Vigoya, M. (2009). La sexualización de la raza y la racialización de la sexualidad en el contexto latinoamericano actual. Revista Latinoamericana de Estudios Familiares, 1(4), 6381.

\section{Breve CV de las autoras}

\section{Gabriela Bard Wigdor}

Becaria de investigación Posdoctoral del Consejo Nacional de Investigaciones Técnicas y Científicas (CONICET). Doctora en Estudios de Género por el Centro de Estudios Avanzados (CEA) de la Universidad Nacional de Córdoba (UNC). Magister en Trabajo Social con mención en Intervención por la Universidad Nacional de Córdoba (UNC), Licenciada en Trabajo Social por la UNC. Trabaja en el Centro de Estudios Avanzados (CIECS), CONICET. Email: gabrielabardw@gmail.com

\section{Gabriela C. Artazo}

Becaria de Investigación CONICET Tipo I. Doctoranda del Doctorado en Cs. Políticas del CEA, Master Internacional en "MERCOSUR y Unión Europea: Diferencias y Similitudes". Licenciada en Trabajo Social. Trabaja en el Instituto Académico Pedagógico de Ciencias Sociales- Universidad de Villa María. Email: artazo2011@hotmail.com.ar 